\title{
OH Maser sources in W49N: probing differential anisotropic scattering with Zeeman pairs
}

\author{
Avinash A. Deshpande ${ }^{1}$, W. M. Goss ${ }^{2}$ and J. E. Mendoza-Torres ${ }^{3}$ \\ ${ }^{1}$ Raman Research Institute, C. V. Raman Avenue, Sadashivanagar, Bangalore, 560080 India \\ email: desh@rri.res.in \\ ${ }^{2}$ National Radio Astronomy Observatory, P.O. Box O, Socorro, NM 87801 USA \\ email: mgoss@aoc.nrao.edu \\ ${ }^{3}$ Instituto Nacional de Astrofisica Optica y Electronica, 72840, Mexico \\ email: mend@inaoep.mx
}

\begin{abstract}
Our analysis of a VLBA 12-hour synthesis observations of the $\mathrm{OH}$ masers in W49N has provided detailed high angular-resolution images of the maser sources, at 1612, 1665 and 1667 MHz. The images, of several dozens of spots, reveal anisotropic scatter broadening; with typical sizes of a few tens of milli-arc-seconds and axial ratios between 1.5 to 3 . The image position angles oriented perpendicular to the galactic plane are interpreted in terms of elongation of electrondensity irregularities parallel to the galactic plane, due to a similarly aligned local magnetic field. However, we find the apparent angular sizes on the average a factor of 2.5 less than those reported by Desai et al., indicating significantly less scattering than inferred earlier. The average position angle of the scattered broadened images is also seen to deviate significantly (by about 10 degrees) from that implied by the magnetic field in the Galactic plane. More intriguingly, for a few Zeeman pairs in our set, we find significant differences in the scatter broadened images for the two hands of polarization, even when apparent velocity separation is less than $0.1 \mathrm{~km} / \mathrm{s}$. Here we present the details of our observations and analysis, and discuss the interesting implications of our results for the intervening anisotropic magneto-ionic medium, as well as a comparison with the expectations based on earlier work.
\end{abstract}

Keywords. masers; ISM: molecules, magnetic fields, individual (W49N); radio lines: ISM

\section{Introduction}

W49N is a well-known and extensively-studied massive star-forming complex in the Milky Way. The $\mathrm{OH}, \mathrm{H}_{2} \mathrm{O}$ masers in this region represent some of the most luminous of such sources in our Galaxy. In our efforts to study intrinsic short time-scale variability in W3OH (Ramachandran et al. 2006; also Laskar et al. in this volume) we were able to estimate and remove possible variability due to interstellar scintillation using the fact that decorrelation bandwidth is larger than line-widths. Such velocity-resolved analysis of $\mathrm{W} 3 \mathrm{OH}$ data has suggested intrinsic variability on 15-20 minute time scale (Ramachandran et al., 2006), whereas W49N data show variations on time-scales of 1 hour or longer (Goss et al., 2007, talk at IAUS 242, Alice Springs).

The W49N complex, in the Galactic direction $l=43^{\circ} .17 ; b=0^{\circ} .01$, is located on the far-side of the Solar circle. Its large distance $(\sim 11.4 \mathrm{kpc})$ and low Galactic latitude together makes this object attractive for studying various propagation effects due to the intervening medium. The interstellar scattering in this case is comparable to that in the Vela direction. In an earlier study, Desai, Gwinn \& Diamond (1994; hereafter DGD94) have found significant anisotropic scattering, attributable to electron-density irregularities that are preferentially elongated in the Galactic plane. In this paper, we describe our 
probe based on the observed manifestations of the interstellar scattering in the W49N direction, compare our results with those from DGD94, and present intriguing cases of differential anisotropic scattering apparent for the two hands of circular polarization.

\section{Our data}

Our visibility data from $12+$ hour synthesis observations with VLBA (BR107) were calibrated \& processed in the standard way using AIPS. The data were self-calibrated, and absolute coordinates are not available due to an absence of phase reference source obervations. The spectral-line image cubes, obtained separately at $1612,1665 \& 1667$ $\mathrm{MHz}$, provided high angular-resolution images of the set of sources for each of the transitions, and for each of the circular polarizations. The imaged angular extent of about 8 or so arcseconds corresponds to a transverse span of $\sim 0.5 \mathrm{pc}$ at the distance of the source, although a majority of the sources are within central 2-arcsecond wide region. As a result of exclusion of the outer 2 antennas of VLBA from Self-Cal and this imaging, our synthesized beam size was compromised to $\sim 20$ mas and $\sim 15$ mas in RA and Declination, respectively (corresponding spatial resolution being $\sim 200 \mathrm{AU}$ and $\sim 150 \mathrm{AU}$ ). Each data set consisting of cleaned and restored images across 240 spectral channels spanning $22 \mathrm{~km} / \mathrm{s}$ velocity range, providing velocity resolution of $\sim 0.1 \mathrm{~km} / \mathrm{s}$, was used to identify discrete maser sources (avoiding cases with significant velocity gradients). For each of clearly identifiable discrete (or isolated) maser sources, JMFIT-based estimates of the deconvolved source shape and size were obtained, in addition to the estimates of the mean location and velocity, along with estimates of the respective uncertainties. The resultant data on a total of 205 sources (most of these at $1665 \& 1667 \mathrm{MHz}$ ) were examined among other things, for positional proximity of LCP and RCP source pairs ( $\leqslant 10$ mas), and a few dozen Zeeman pairs were thus identified. Figure 1 shows the distribution of all of the discrete sources in the RA-Dec plane, where the size of the symbol is proportional to the mean velocity associated with the observed line.

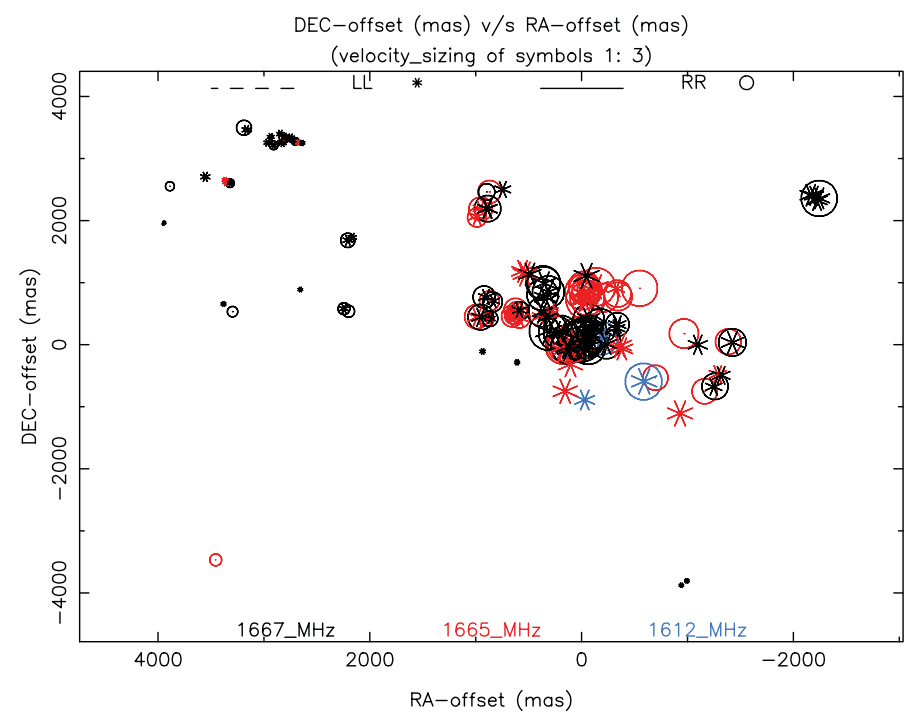

Figure 1. The observed distribution of W49N OH maser sources in RA-Dec. The symbol sizes are scaled proportional to the velocity (which are in the range +2 to $+21 \mathrm{~km} / \mathrm{s}$ ). The velocity-position correlation, and the implied bipolar nature, is apparent from the majority of the sources in the sample. 


\section{Anisotropic scattering: apparent source sizes and shapes}

Scatter-broadened shapes and orientations of the maser spots provide valuable information on the nature of the scattering medium. The elongations or the axial ratios (ratio of major to minor axis of the best-fit ellipse) are found in the range 1.5 to 3 , indicating significant anisotropic scattering, consistent with the finding of DGD94. However, we find the overall apparent sizes of the sources to be significantly smaller (by a factor of $\geqslant 2$ ) than those reported by DGD94. Our size estimates are nonetheless consistent with the suitably scaled values of the scatter-broadening of $\mathrm{H}_{2} \mathrm{O}$ masers reported by Gwinn (1994). Possible reasons for what appears to be an overestimation of the sizes in DGD94 are unclear, though remain intriguing. One of the most important aspects discussed by DGD94 (also see references therein) was that the apparent image shapes resulting from anisotropic diffraction are expected to have elongation orthogonal to that of the scattering irregularities. A presence of a magnetic field would induce such anisotropy in the electron-density irregularities; then the implied field direction would be perpendicular to the resultant image position angle. Based on their limited sample, of the apparent image shapes for 27 spectral components from 6 sources, DGD94 had suggested that the apparent anisotropy is induced by a magnetic field in and parallel to the Galactic plane.

In Figure 2, we present a distribution of our estimates of the position angles for our significantly ( $\sim 30$ times) larger sample. Some random spread in PA is apparent in both, our and DGD94's, reported PA values, and such a spread is not unexpected. However, we find that our PA distribution has a significant offset from the PA of $\sim 117$ degrees, the expected PA if the density irregularities were to be elongated parallel to the Galactic plane. Our sample gives a mean PA of $107 \pm 3$ degrees, implying a significant mean deviation, of $\sim 10$ degrees. The DGD94 sample may have been too limited to make such a offset detectable. What might be the reason for this systematic offset? In this context,

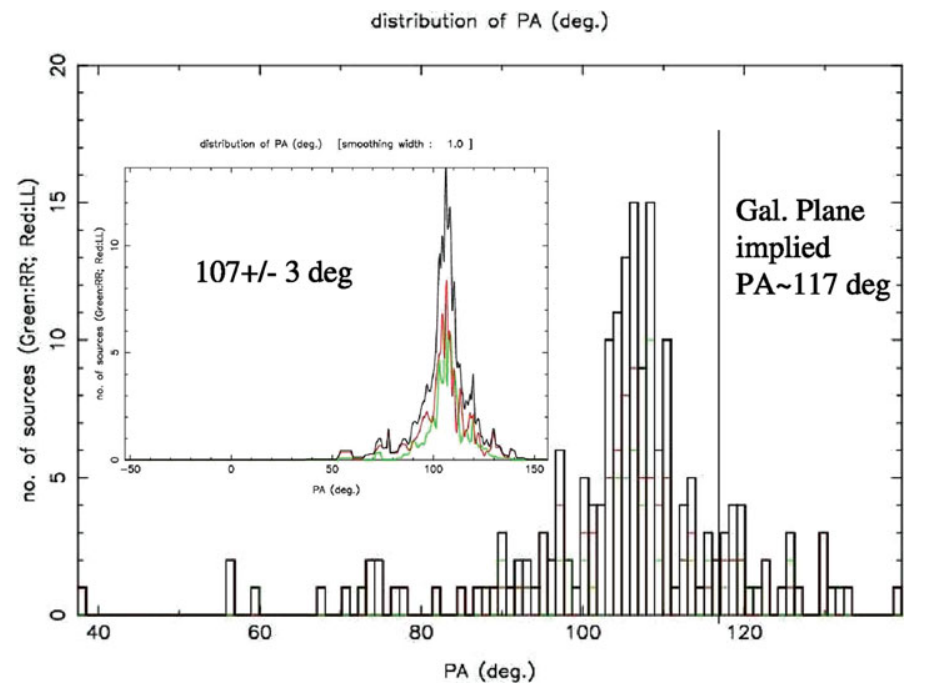

Figure 2. Distribution of the position angles (PA) of the scatter-broadened images. The main panel shows the histogram of the PA values for the set of $\mathrm{OH}$ maser sources in W49N. The vertical line at 117 degrees, shown for reference, marks the mean PA expected from the anisotropic scattering due to electron density irregularities, if these were to be elongated parallel to the Galactic plane. The inset shows the distribution of PAs after accounting for uncertainties in individual measurements (green and red profile correspond to LCP and RCP, respectively). This distribution is used to estimate the mean PA of the observed scatter-broadened images (107 \pm 3 degrees). 
a closer look at the magnetic field structure along the W49N direction would be instructive. Interestingly, our sight-line to $\mathrm{W} 49 \mathrm{~N}$ is through the well-known North Polar Spur feature. From the study, by Wolleben (2007) and others, of the North Polar Spur, it is evident that significantly different magnetic field structure as well as enhanced scattering would be expected for the medium within half a kpc of the Sun. We note that the field orientation in this region, corresponding to the NPS, might be at a large inclination, if not almost orthogonal, to the Galactic plane. Also, given that the scattering medium closer to the observer is expected to make relatively higher contribution to the angular broadening (e.g., Gwinn et al. 1993, Deshpande and Ramachandran 1998), the above mentioned PA deviation can be caused by the more local scattering in the NPS region, with density anisotropy at a possibly large angle with respect to the Galactic plane. Careful modelling of the magneto-ionic medium would reveal the relative strengths and sense of anisotropic scattering, implied magnetic field strengths, for the different regions along the sight-line. Details and results of such modelling will be discussed elsewhere.

\section{Differential Anisotropic scattering: probe with Zeeman pairs}

As mentioned earlier, a few dozen Zeeman pairs are identified from our large sample of sources, using positional-proximity criterion. These Zeeman pairs allow us to probe yet unobserved aspect of scattering caused by the magneto-ionic component of the interstellar medium. A magneto-ionic medium would, in principle, render different refractive indices for the two hands of circular polarization. Diffractive scintillation and scatterimage shapes should therefore differ for LHC, RHC due to line-of-sight component of the magnetic field in the intervening medium. Hence scattering-dominated images of even a randomly polarized source might show circular polarization in unmatched parts of the images, when the Faraday rotation is significant. Macquart \& Melrose (2000) indeed consider this possibility, but estimate the effect to be too small (circular fraction $\sim 10^{-8}$ ) to be observable. Contrary to that expectation, the scatter-broadened images of some of the W49N OH maser sources seem to significantly differ in L\&R-hand circular polarizations, i.e. within a given Zeeman pair ! For the rest of the Zeeman pairs, the PA differences are either small or within the respective uncertainties. Figure 3 shows the observed differences in the image shape parameters for a subset of our sample of Zeeman pairs. The subset includes only those cases for which significant difference $(\geqslant 6 \sigma)$ in image PA is observed, when compared to corresponding uncertainty $\sigma$. Although there are only a few such Zeeman pairs, the PA differences range between 6 to 30 degrees. Difference in the line-velocities within most of these pairs, and hence in frequencies, is too small to account for the apparent differential scattering. Position differences are also within a few mas, and are unlikely to contribute to the observed PA differences.

To explore the issue further, we have made preliminary attempts to simulate magnetoionic medium with a mild anisotropy. A random column-density distribution of free electrons, following a power-law spatial spectrum (with Kolmogorov index $-11 / 3$ ), is used to simulate a 2 -d scattering screen across a transverse extent of a few Fresnel scales. For simplicity, a uniform magnetic field is assumed, so that the phase screens for the two circular polarizations are merely scaled versions of each other. The net circular polarization, if viewed with coarse resolution would be negligible, consistent with the conclusion of Macquart \& Melrose (2000). However, when viewed with 10 or so milliarcsecond resolution, these preliminary simulations do indeed reveal noticeable differential diffractive effects, and hence the differing shapes and sizes of scatter-broadened images, for the two circular polarizations. Of course, more detailed simulations, with thick screens, will be needed to assess these issues in detail. 
pair_PA_diff (deg) v/s pair_axratio_diff for Zeeman pairs

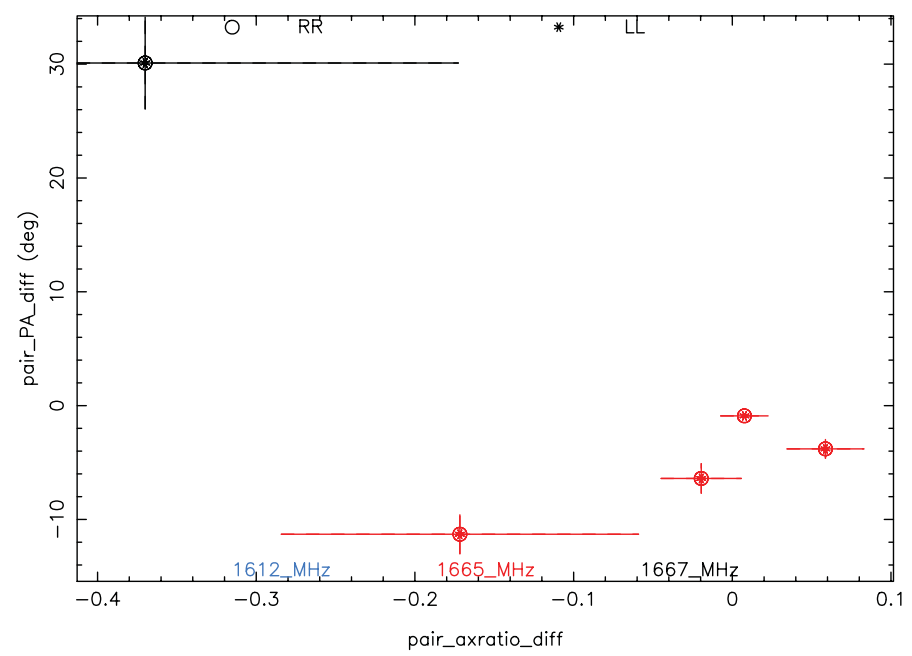

Figure 3. The PA differences within Zeeman pairs versus the respective differences in axial ratios for the cases where significant PA differences are apparent. The error-bars indicate $\pm 1-\sigma$ uncertainties. All the axial ratios are all consistent with equal values for the two hands of circular polarization.

\section{Summary}

The $\mathrm{OH}$ maser sources in W49N do show anisotropic scattering, but the apparent scatter broadening is much less than reported earlier. The position angles of the source images deviate significantly from the value expected if scattering density irregularities were to be "stretched" due to magnetic field strictly aligned parallel to the Galactic plane, indicating significant scatter-broadening contribution from differently aligned density irregularities, possibly associated with the North Polar Spur. Some of our data also reveal differential scattering during propagation of the two circular polarizations, caused most likely by Faraday rotation. Through various aspects discussed above, the attractive, but yet unexplored, potential of the high-resolution maser observations for probing the intervening magneto-ionic medium is certainly evident.

\section{Acknowledgements}

It is a pleasure to acknowledge the contributions from R. Ramachandran and Sarah Streb at different stages of the reported work. The National Radio Astronomy Observatory is a facility of the Natiional Science Foundation operated under a cooperative agreement by Associated Universities, Inc.

\section{References}

Desai, K. M., Gwinn, C. R., \& Diamond, P. J. 1994, Nature, 372, 754 (DGD94)

Deshpande, A. A. \& Ramachandran, R. 1998, MNRAS, 300, 577

Gwinn, C. R., Bartel, N., \& Cordes, J. M. 1993, ApJ, 410, 673

Gwinn, C. R. 1994, ApJ, 429, 253

Macquart, J.-P. \& Melrose, D. B. 2000, ApJ, 545, 798

Ramachandran, R., Deshpande, A. A., \& Goss, W. M. 2006, ApJ, 653, 1314

Wolleben, M. 2007, ApJ, 664, 349 